\title{
MOF Decomposition and Introduction of Repairable Defects Using a Photodegradable Strut
}

\author{
Jingjing Yan, ${ }^{[a]}$ John C. MacDonald, ${ }^{[a]}$ Alex R. Maag, ${ }^{[b]}$ François-Xavier Coudert ${ }^{[c]}$ and Shawn \\ Burdette ${ }^{*[a]}$
}

\begin{abstract}
Photoswitchable components can modulate the properties of metal organic frameworks (MOFs); however, photolabile building blocks remain underexplored. A new strut NPDAC (2-nitro-1,4phenylenediacetic acid) that undergoes photodecarboxylation has been prepared and incorporated into a MOF using post-synthetic linker exchange (PSLE) from the structural analogue containing PDAC ( $p$-phenylenediacetic acid). Irradiation of NPDAC-MOF leads to MOF decomposition and concomitant formation of amorphous material. In addition to complete linker exchange, MOFs containing a mixture of PDAC and NPDAC can be obtained through partial linker exchange. In NPDAC30-MOF which contains approximately $30 \%$ NPDAC, the MOF retains crystallinity after irradiation, but the MOF contains defect sites consistent with loss of decarboxylated NPDAC linkers. The defect sites can be repaired by exposure to additional PDAC or NPDAC linkers at a much faster rate than the initial exchange process. The photoremoval and replacement process may lead to a more general approach to customizable MOF structures.
\end{abstract}

\section{Introduction}

The large void spaces and high surface areas within metalorganic frameworks (MOFs) make them appealing for a wide variety of applications in storage, ${ }^{[1]}$ sequestration [2] and separations. ${ }^{[3]}$ With the notable exception of recent interest in flexible systems, ${ }^{[4]}$ most MOFs applications take advantage of the rigid structures to achieve the desired properties; however, the scope of possible applications would be expanded if MOFs could be designed to respond to external stimuli. Incorporating azobenzene ligands onto the side chains of struts can create photoresponsive MOFs. Azobenzene cis $\leftarrow$ trans photoisomerization provides a technique to block MOF channels $;{ }^{[5]}$ however, azobenzene isomerization is nearly ${ }^{[6,7]}$ or

[a] Dr. J. Yan, Prof. Dr. J. C. MacDonald, Prof. Dr. S. C. Burdette Department of Chemistry and Biochemistry Worcester Polytechnic Institute

100 Institute Road, Worcester, MA 01609-2280 (USA)

E-mail: scburdette@wpu.edu

[b] Department of Chemical Engineering

Worcester Polytechnic Institute

100 Institute Road, Worcester, MA 01609-2280 (USA)

[c] Prof. Dr. François-Xavier Coudert

Chimie ParisTech, PSL

University, CNRS, Institut de Recherche de Chimie

Paris, 75005 Paris, France completely $\left[{ }^{[,}, 9\right]$ restricted when the ligand is utilized as a structural support. Moreover, photochromic diarylethene derivatives have been incorporated as MOF struts, and local framework movement can be achieved by diarylethene ring opening and closing,, $10-12$ but the framework change is minimal and the MOF skeleton is retained. Alternatively, photolabile protecting groups on side chains can be removed to control channel access, $\left.{ }^{[13}, 14\right]$ but the extent to which strut photolysis has been explored is limited.

MOFs are susceptible to degradation under acidic conditions, or when exposed to strongly coordinating molecules, due to linker protonation and displacement respectively. Although these and other processes of structural breakdown are impediments in some fields, MOF decomposition can be exploited for molecular release in applications such as drug delivery. ${ }^{[15]}$ Most MOFs designed for drug delivery are engineered to respond to the acidic compartments in the cell cytoplasm or in lysosomes. ${ }^{[16,}$ 17] In the absence of $\mathrm{pH}$ changes however, the options for triggered release of guest from MOFs are restricted. As with MOF side chains, the most convenient approach to controlled degradation would be light-mediated processes.

Light degradable materials have been studied extensively, 18 20] but there are limited examples of MOF photodecomposition. While complexes that release metal ions in response to light are relatively common[21, 22] including photocaged complexes that release zinc upon decarboxylation, [23] the analogous photochemistry has not been explored extensively in MOFs. Photodecarboxylation in a benzothiadiazole-derived MOF led to detectable structural changes and differences in gas adsorption although the experimental data for the proposed behavior not definitive, ${ }^{24]}$ and an azobenzene-containing MOF reportedly degrades upon irradiation;[25] however, irradiation only accelerates the basal rate of decomposition. Local and dynamic structural modulation upon irradiation has been reported with MOFs $^{[6]}$ and COFs ${ }^{[7]}$ containing photoswitchable struts; however, direct evidence for the changes are limited. Inspired by our earlier studies on molecule release triggered by photodecarboxylation of a capping on MOF surface, $\underline{[26]}$ we envisioned incorporating photolabile dicarboxylates as struts to facilitate photodegradation of MOFs. Although benzoic acids typically used in MOF synthesis are quite photostable, we hypothesized that photoactive phenylacetic acids derivatives could be incorporated in MOF structures and provide access to degradable materials.

\section{Experimental Section}

General Procedures. All reagents were purchased and used without further purification. Thin-layer chromatography (TLC) was performed on 
silica (200-400 mesh). TLCs were developed by using mixture of dichloromethane (DCM) and methanol (MeOH). ${ }^{1} \mathrm{H}$ and ${ }^{13} \mathrm{C}$ NMR spectra were recorded with a $500 \mathrm{MHz}$ Bruker Biospin NMR instrument. FT-IR spectra were recorded using Bruker Vertex70 Optics FT-IR spectrometer equipped with a Specac Golden Gate attenuated total reflection (ATR) accessory by collecting 256 scans over a scan range from 4000 to $600 \mathrm{~cm}$ 1 at $4 \mathrm{~cm}^{-1}$ resolution. Elemental microanalyses for $\mathrm{C}, \mathrm{H}$, and $\mathrm{N}$ were performed by Micro Analysis Inc. (Wilmington DE). Thermogravimetric analysis (TGA) measurements were carried out on a TA Instruments $\mathrm{Hi}-$ Res TGA 2950 Thermogravimetric Analyzer from room temperature to $800^{\circ} \mathrm{C}$ under nitrogen atmosphere at a heating rate of $10^{\circ} \mathrm{C} / \mathrm{min}$. LC/MS was carried on a Single Quadruple, Agilent Technologies 1200 series LC system. High resolution mass spectra were obtained at the University of Notre Dame mass spectrometry facility using microTOF instrument operating in positive ionization mode. Melting-point information was obtained using a Hydrothermal Mel-Temp instrument.

2-Nitro-1,4-phenylenediacetic acid (NPDAC, 2). Concentrated sulfuric acid $(5 \mathrm{~mL})$ and $p$-phenylenediacetic acid $(0.300 \mathrm{~g}, 1.54 \mathrm{mmol})$ were combined at $0{ }^{\circ} \mathrm{C}$, and concentrated nitric acid $(0.5 \mathrm{~mL})$ was added dropwise to the mixture. After stirring in the dark for $1 \mathrm{~h}$, the reaction mixture was added dropwise to ice $(200 \mathrm{~g})$, and the product was extracted into EtOAc $(2 \times 100 \mathrm{~mL})$. The combined organic layers were washed with saturated $\mathrm{NaCl}(2 \times 100 \mathrm{~mL})$, dried over $\mathrm{Na}_{2} \mathrm{SO}_{4}$ and the solvent was removed to yield the product as a light-yellow powder without further purification $\left(0.280 \mathrm{~g}, 76.1 \%\right.$ yield). TLC $R_{f}=0.41$ (silica, 6.5:1, $\mathrm{DCM} / \mathrm{MeOH}) . \mathrm{Mp}=246-248{ }^{\circ} \mathrm{C} .{ }^{1} \mathrm{H}$ NMR $\left(500 \mathrm{MHz}, \mathrm{DMSO}-\mathrm{d}_{6}\right) \delta 12.55$ $(\mathrm{s}, 2 \mathrm{H}), 8.01(\mathrm{~s}, 1 \mathrm{H}), 7.59(\mathrm{~d}, J=7.88 \mathrm{~Hz}, 1 \mathrm{H}), 7.48(\mathrm{~d}, J=7.88 \mathrm{~Hz}, 1$ H), $3.97(\mathrm{~s}, 2 \mathrm{H}), 3.75(\mathrm{~s}, 2 \mathrm{H}) .{ }^{13} \mathrm{C}$ NMR (125 MHz, DMSO-d 6 ) $\delta 172.6$, 171.9, 148.8, 136.5, 135.5, 133.9, 129.3, 126.1. FT-IR (neat, $\mathrm{cm}^{-1}$ ) 2966.7, $2647.5,2549.6,1696.4,1529.3,1409.8,1351.7,1289.7,1231.6,1202.9$, $1144.8,1079.5,916.3,887.0,825.6,810.6,763.6,738.1,676.8$. HRMS (+ESI) calculated for $\mathrm{MNa}^{+}$262.0322, observed 262.0341 .

[Zn(PDAC)(4,4'-bipyridyl) $\left.\cdot \mathrm{CH}_{3} \mathrm{OH}\right]_{n}$ (PDAC-MOF). PDAC-MOF was prepared at room temperature by modifying the reported solvothermal synthesis. [27] Sodium hydroxide $(8.00 \mathrm{mg}, 0.200 \mathrm{mmol})$ was added to a solution of $p$-phenylenediacetic acid $(19.4 \mathrm{mg}, 0.100 \mathrm{mmol})$ and 4,4 'bipyridine (15.6 mg, $0.100 \mathrm{mmol}$ ) in $1 \mathrm{~mL}$ of $\mathrm{MeOH} / \mathrm{H}_{2} \mathrm{O}(4: 1)$. The solution was layered with $\mathrm{MeOH}(5 \mathrm{~mL})$ to induce slow mixing with a third layer of $\mathrm{MeOH}(1 \mathrm{~mL})$ containing zinc nitrate tetrahydrate $\left(\mathrm{Zn}\left(\mathrm{NO}_{3}\right)_{2} \cdot 6 \mathrm{H}_{2} \mathrm{O} 226\right.$ $\mathrm{mg}, 0.100 \mathrm{mmol}$ ) for $5 \mathrm{~d}$ to yield colorless needles ( $33.0 \mathrm{mg}$, yield $74.1 \%$ ). Anal. Calc. for $\mathrm{C}_{21} \mathrm{H}_{20} \mathrm{~N}_{2} \mathrm{O}_{5} \mathrm{Zn}$ : C, 56.53; $\mathrm{H}, 4.49 ; \mathrm{N}, 6.28 \%$. Found: C, $56.21 ; \mathrm{H}, 4.62 ; \mathrm{N}, 6.35 \%$. FT-IR (neat, $\mathrm{cm}^{-1}$ ) 3433.9, 3348.6, 3034.5, 2922.3, 1652.6, 1586.2, 1562.8, 1514.0, 1490.6, 1435.9, 1402.8, 1324.6, 1297.2, 1277.7, 1217.2, 1201.6, 1170.3, 1145.1, 1103.9, 1072.6, 1047.4, 1031.7, 1008.3, 953.6, 863.7, 844.4, 818.9, 770.1, 732.9, 703.7, 664.6, 631.5. TGA shows a $15.7 \%$ weight loss between $23-40{ }^{\circ} \mathrm{C}$, which may correspond to absorbed solvent. Decomposition occurs at $135{ }^{\circ} \mathrm{C}$. Brunauer-Emmett-Teller shows a surface area of $6.6 \mathrm{~m}^{2} \mathrm{~g}^{-1}$ for $\mathrm{N}_{2}$ molecules.

[Zn(NPDAC)(4,4'-bipyridyl) $\left.\mathrm{CH}_{3} \mathrm{OH}\right]_{\mathrm{n}}$ (NPDAC-MOF). NPDAC $(215 \mathrm{mg}$ $0.900 \mathrm{mmol})$ and $\mathrm{NaOH}(72.0 \mathrm{mg}, 1.80 \mathrm{mmol})$ were dissolved in $10 \mathrm{~mL}$ $\mathrm{MeOH}$ to prepare a $0.090 \mathrm{M}$ NPDAC sodium salt solution. Crystalline PDAC-MOF $(20.0 \mathrm{mg}, 45.0 \mu \mathrm{mol})$ was immersed in $5 \mathrm{~mL} \mathrm{MeOH}$, and $5 \mathrm{~mL}$ of NPDAC sodium salt solution $(0.090 \mathrm{M})$ was added. The mixture was kept in dark for $7 \mathrm{~d}$ to yield colorless needles suitable for $\mathrm{X}$-ray analysis (16.7 mg, yield 83.2\%). FT-IR (neat, $\mathrm{cm}^{-1}$ ) 2918.2, 2683.5, 1629.2, 1609.7, 1580.5, 1527.6, 1494.5, 1416.3, 1342.3, 1269.9, 1219.1, 1146.9, 1072.6, 1047.4, 1014.3, 924.4, 897.0, 863.7, 811.0, 783.7, 721.1, 690.1, 637.2. The ligand composition of the resulting MOF crystals was determined by ${ }^{1} \mathrm{H}$ NMR after digesting the crystals with $\mathrm{D}_{2} \mathrm{SO}_{4}$ in DMSO- $\mathrm{d}_{6}$, which show $100 \%$ of the original PDAC was replaced by NPDAC. TGA shows a $16.5 \%$ weight loss between $23-46{ }^{\circ} \mathrm{C}$, which may correspond to absorbed solvent. Decomposition occurs at $112{ }^{\circ} \mathrm{C}$. Brunauer-Emmett-Teller shows a surface area of $6.3 \mathrm{~m}^{2} \mathrm{~g}^{-1}$ for $\mathrm{N}_{2}$ molecules.

NPDAC30-MOF. PDAC-MOF crystals $(20.0 \mathrm{mg}, 45.0 \mu \mathrm{mol})$ were immersed in $5 \mathrm{~mL} \mathrm{MeOH}$, and combined with $1.5 \mathrm{~mL}$ of NPDAC sodium salt solution $(0.090 \mathrm{M})$ and $3.5 \mathrm{~mL}$ additional $\mathrm{MeOH}$. The mixture was kept in dark for $7 \mathrm{~d}$ to induce linker exchange. The ligand composition of the resulting MOF crystals was determined by ${ }^{1} \mathrm{H}$ NMR after digesting the crystals with $\mathrm{D}_{2} \mathrm{SO}_{4}$ in DMSO- $\mathrm{d}_{6}$, which show approximately $30 \%$ of the original PDAC was replaced by NPDAC.

Gas sorption. Gas sorption was performed using an ASIQ iQ Quantachrome Instrument by adding degassed of PDAC-MOF, NPDACMOF or photolyzed NPDAC30-MOF $(30 \mathrm{mg})$ and increasing the temperature at $2{ }^{\circ} \mathrm{C} \mathrm{min}{ }^{-1}$ with 15 -min temperature holds at $60,80,100$ and $120{ }^{\circ} \mathrm{C}$ before increasing to $150{ }^{\circ} \mathrm{C}$, where the temperature was maintained for 11 hours. Nitrogen was dosed as the adsorbate into the sample cell cooled with liquid $\mathrm{N}_{2}$ and 50 isothermal $\mathrm{P} / \mathrm{P}_{0}$ points ranging from $6 \times 10^{-6}$ to 0.95 followed by 15 desorption points between $P / P_{0}$ of 0.95 to 0.1 were obtained. Each surface area was determined by applying the Brunauer-Emmett-Teller (BET) method to model the adsorption isotherm between $\mathrm{P} / \mathrm{P}_{0}$ of 0.15 to 1 .

X-ray Crystallography. Structural analysis was carried out in the X-Ray Crystallographic Facility at Worcester Polytechnic Institute. Crystals were glued on tip of a glass fiber or were covered in PARATONE oil on $100 \mu \mathrm{m}$ MiTeGen polyimide micromounts and were mounted on a Bruker-AXS APEX CCD diffractometer equipped with an LT-II low temperature device. Diffraction data were collected at room temperature using graphite monochromated Mo-Ka radiation $(\lambda=0.71073 \AA)$ using the omega scan technique. Empirical absorption corrections were applied using the SADABS program. ${ }^{[28]}$ The unit cells and space groups were determined using the SAINT+ program. [28] The structures were solved by direct methods and refined by full matrix least-squares using the SHELXTL program. [29] Refinement was based on $\mathrm{F}^{2}$ using all reflections. All nonhydrogen atoms were refined anisotropically. Hydrogen atoms on carbon atoms were all located in the difference maps and subsequently placed at idealized positions and given isotropic $U$ values 1.2 times that of the carbon atom to which they were bonded. Hydrogen atoms bonded to oxygen atoms were located and refined with isotropic thermal parameters. Mercury 3.1 software and Diamond Version 3.1d were used to examine the molecular structure. Relevant crystallographic information is summarized in Table 1, and the 50\% thermal ellipsoid plots are shown in Figure $1 \mathrm{~A}$.

Powder X-ray diffraction. PXRD data were collected on a Bruker-AXS D8-Advance diffractometer using $\mathrm{Cu}$-Ka radiation with $\mathrm{X}$-rays generated at $40 \mathrm{kV}$ and $40 \mathrm{~mA}$. Bulk samples of crystals were placed in a $20 \mathrm{~cm} \times 16$ $\mathrm{cm} \times 1 \mathrm{~mm}$ well in a glass sample holder, and scanned at RT from $3^{\circ}$ to $50^{\circ}(2 \theta)$ in $0.05^{\circ}$ steps at a scan rate of $2^{\circ} / \mathrm{min}$. Simulated PXRD patterns from single crystal data were compared to PXRD patterns of PDAC-MOF and NPDAC-MOF to confirm the uniformity of the crystalline samples.

General Spectroscopic Methods. All aqueous solutions were prepared from Millipore (BiopakTM Ultrafiltration Cartridge) water. All organic solutions were prepared using spectroscopic grade solvents. UV-vis absorption spectra were obtained by taking sample solutions in $1.0 \mathrm{~cm}$ quartz cuvette at $23{ }^{\circ} \mathrm{C}$ with total volumes kept at $2 \mathrm{~mL}$ or $3 \mathrm{~mL}$, and recorded on Thermo Scientific Evolution 300 UV-vis spectrometer with inbuilt Cary winUV software. Photolysis was carried at $23{ }^{\circ} \mathrm{C}$ in $1.0 \mathrm{~cm}$ quartz cuvette illuminated by 3 W UV LED (Mouser Electronics, $365 \mathrm{~nm}$, $200 \mathrm{~mW}$ ) powered by a $700 \mathrm{~mA}$ LuxDrive FlexBox using a variable DC source set at 12 VDC. Rate of photolysis and photoproducts were 
analyzed using LC/MS (Single Quadrupole, Agilent Technologies) by monitoring at changes at $277 \mathrm{~nm}$.

Quantum Efficiency and Photoproducts Determination. A $2 \mathrm{~mL}$ solution of NPDAC $(15 \mathrm{mM})$ in $\mathrm{MeOH}\left(5 \% \mathrm{H}_{2} \mathrm{O}\right)$ was prepared from a 45 $\mathrm{mM}$ stock solution and exposed to a LED UV irradiation for $60,180,360$, and $600 \mathrm{~s}$. A fresh $2 \mathrm{~mL}$ solution was used for each time interval irradiation, and $100 \mu \mathrm{L}$ of ketoprofen in $\mathrm{MeOH}(100 \mathrm{mM})$ was added as internal standard before subjecting samples to LC-MS analysis. The quantum efficiency was calculated following established procedures. [29] The photoproducts were confirmed using HPLC and ${ }^{1} \mathrm{H}$ NMR analysis. All the samples were eluted using an isocratic mixture of $95: 5 \mathrm{CH}_{3} \mathrm{CN}: \mathrm{H}_{2} \mathrm{O}$ containing $0.1 \%$ formic acid at a flow rate of $0.3 \mathrm{~mL} / \mathrm{min}$. The identity of individual peaks that are labeled from 2-5 in the HPLC traces in Figure 3 were identified by $\mathrm{m} / \mathrm{z}$ values in the mass spectrum. Bulk photolysis of NPDAC was carried out by dissolving NPDAC (23.9 mg, $0.100 \mathrm{mmol})$ in 2 $\mathrm{mL} \mathrm{D}_{2} \mathrm{O}$ containing $\mathrm{NaOH}(8.0 \mathrm{mg}, 0.20 \mathrm{mmol})$. The solution was irradiated with $365 \mathrm{~nm}$ light for $35 \mathrm{~min}$. The $\mathrm{D}_{2} \mathrm{O}$ mixture was analyzed by ${ }^{1} \mathrm{H}$ NMR spectroscopy, and then extracted successively with $2 \mathrm{~mL}$ hexanes and 2 $\mathrm{mL} \mathrm{DCM}$, and the combined organic phase was removed. The combined organic-soluble extracts were dissolved in $\mathrm{CDCl}_{3}$ and the ${ }^{1} \mathrm{H} \mathrm{NMR}$ was recorded.

Photolysis of NPDAC-MOF. NPDAC-MOF $(5.00 \mathrm{mg}, 11.2 \mu \mathrm{mol})$ was immersed in $\mathrm{MeOH}(2 \mathrm{~mL})$, kept in dark for $2 \mathrm{~h}$, and UV-vis absorption spectra was recorded. The mixture was exposed to $365 \mathrm{~nm}$ of light for $2 \mathrm{~h}$ in total, the first $1 \mathrm{~h}$ without stirring and the second hour with stirring. After 10 min of irradiation, the mixture was removed to the dark for 30 min before measuring the absorbance of the supernatant liquid. This process was repeated after total irradiation times of $20,30,45$ and $60 \mathrm{~min}(3.5 \mathrm{~h}$ elapsed). After irradiation, the solution was filtered through glass wool, and LC-MS analysis was performed on the filtrate using ketoprofen as an internal standard. The sample was eluted using an isocratic mixture of 95:5 $\mathrm{CH}_{3} \mathrm{CN}: \mathrm{H}_{2} \mathrm{O}$ containing $0.1 \%$ formic acid at a flow rate of $0.3 \mathrm{~mL} / \mathrm{min}$, and the absorbance at $277 \mathrm{~nm}$ was monitored.

Defects Creation and MOF Repair. NPDAC30-MOF $(20 \mathrm{mg})$ was as immersed in $\mathrm{MeOH}(2 \mathrm{~mL})$. The mixture was irradiated $(365 \mathrm{~nm})$ with stirring for $2 \mathrm{~h}$. The resulting material was isolated, rinsed with $\mathrm{MeOH}$, and divided into two equal portions. The two samples were immersed in $5 \mathrm{~mL}$ $\mathrm{MeOH}$ containing $135 \mu \mathrm{mol}$ PDAC or $1.5 \mathrm{~mL}$ NPDAC sodium salt solution $(0.090 \mathrm{M})$ combined with $3.5 \mathrm{~mL} \mathrm{MeOH}$ for $1 \mathrm{~d}$. The materials were isolated and crystallinity was verified by PXRD. The ligand composition of the resulting MOF crystals was determined by ${ }^{1} \mathrm{H}$ NMR after digesting the crystals with $\mathrm{D}_{2} \mathrm{SO}_{4}$ in DMSO-d6.

Computational methods. Quantum chemistry calculations were performed using a density functional theory (DFT) approach on the periodic structures with full accounting for symmetry and all-electron localized basis sets $[30,31]$ using the CRYSTAL14 software. ${ }^{[32]}$ Calculations were performed at the generalized-gradient approximation (GGA) level, with the PBEsol exchange-correlation functional. ${ }^{[33]}$ Long-range dispersion interactions were taken into account using the Grimme "D2" dispersion correction scheme. ${ }^{[34]}$ The $k$-point mesh was generated using the Monkhorst-Pack method, with a $2 \times 2 \times 2$ supercell. Given the layered nature of the structure, we performed each energy minimization with respect to atomic positions only, with fixed unit cell parameters, and varied the value of the a lattice parameter in an external loop. Representative input files are available online in the data repository at https://github.com/fxcoudert/citable-data..

\section{Results and Discussion}

Since multicarboxylates, specifically dicarboxylate struts like 1,4-benzenedicarboxylic acid (BDC), are nearly ubiquitous in MOFs, we envisioned designing a light-reactive dicarboxylate ligand as the photoreactive MOF unit. We recently exploited the photochemistry of meta-nitrophenylacetic acid ( $m$-NPAA) derivatives to design zinc photocages ${ }^{[23]}$ and photocapping groups, ${ }^{26]}$ so we hypothesized that a similar functional group could be adapted to construct the desired photodegradable strut. Although much less common than BBC, para-phenylenediacetic acid (PDAC) has been used to construct MOFs with ${ }^{[35,36]}$ and without bipyridine groups. ${ }^{[27]}$ In contrast to BDC as well as other related benzoic acids, the methylene spacer between the aryl and carboxylic acid groups means that PDAC is more flexible, and the diacid strut must adopt a non-planar carboxylate-carboxylate arrangement between metal nodes. This structural flexibility likely explains the limited number of MOFs reported using PDAC-like ligands.

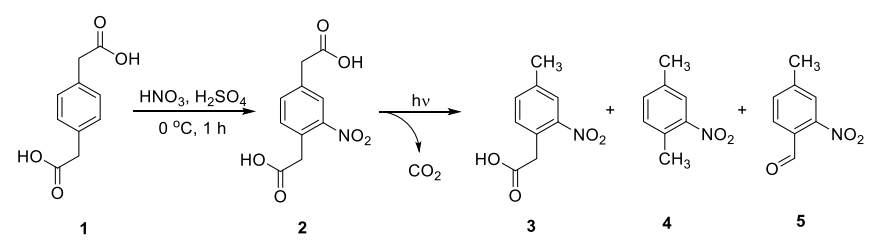

Scheme 1. Synthesis and photolysis of 2 , showing the three major photoproducts, 4-methyl-2-nitrophenylacetic acid (3), 2-nitro-p-xylene (4) and 4methyl-2-nitrobenzaldehyde (5)

Selective nitration of PDAC yielded the desired photoactive linker NPDAC in $76 \%$ yield, since mono-nitration deactivates the ring to further electrophilic aromatic substitution reactions (Scheme 1). Upon exposure to light, NPDAC decomposes to provide a mixture of photoproducts that includes 4-methyl-2nitrophenylacetic acid (3) with $\mathrm{m} / \mathrm{z} 194.1$, 2-nitro- $p$-xylene (4) with $\mathrm{m} / \mathrm{z} 302.0$ as a dimer ion, and 4-methyl-2-nitrobenzaldehyde (5) with $\mathrm{m} / \mathrm{z}$ 164.0, which was determined by LCMS analysis and verified by NMR. Bulk photolysis of NPDAC in $\mathrm{D}_{2} \mathrm{O}$ also was monitored by ${ }^{1} \mathrm{H}$ NMR. After $35 \mathrm{~min}$ of irradiation, the ${ }^{1} \mathrm{H}$ NMR shows a mixture of NPDAC and one major photoproduct, 4methyl-2-nitrophenylacetic acid (3) that is produced by the photodecarboxylation characteristic of $m$-NPAA compounds. $[37,38]$ Several minor unidentifiable photoproducts also are observable. During the irradiation, the clear yellowish solution became opaque, which indicates the possible formation of hydrophobic photoproducts. After extracting the mixture with hexane and then DCM, two additional photoproducts were recovered Photoproduct $\mathbf{4}$ corresponds to a second photodecarboxylation at the ortho position. While decarboxylation ortho-NPAA was anticipated, ${ }^{[37]}$ the exact pathway that leads to the aldehyde 5 remains unclear. Under aerobic conditions however, ortho- and para-nitrotoluene derivatives are susceptible to oxidation, and the photolysis conditions are amenable to radical formation, which could account for the observed photoproduct. ${ }^{[39-42]}$ Phenylacetic acids also can be converted directly to benzaldehyde derivatives with various reagents, ${ }^{[43-45]}$ so the presence of $\mathbf{5}$ is unremarkable. 
A photolysis quantum yield ( $\left.\Phi_{\text {photolysis }}\right)$ of $33 \%$ was calculated by quantifying the disappearance of NPDAC over the course of the reaction using LCMS.

The NPDAC ligand exhibited the desired photodecarboxylation chemistry, but initially we were unable to access a MOF due to apparent decomposition of NPDAC strut during the solvothermal synthesis conditions used to prepare PDAC-MOF. [27, 35] In order to avoid thermal decomposition, we examined solvent layering techniques to prepare PDAC-MOF crystals as a facile alternative to high temperature conditions. The PDAC-MOF framework we obtained at room temperature exhibits an identical semi-pillared paddle-wheel (sppw) structure as previously reported, but with an improved $R$ value. ${ }^{[35]}$ Solvent layering techniques however, failed to produce crystalline material when PDAC was replaced with NPDAC.

Owing to the failure of direct synthetic methods, we explored post-synthetic linker exchange (PSLE) as a different strategy to prepare a photodegradable MOF. PSLE has been applied successfully to prepare MOFs that resist de novo syntheses, ${ }^{[46-48]}$ and as a strategy to prevent framework interpenetration. $\left.{ }^{[49}, 50\right]$ When MOFs are introduced into a concentrated solution of the new ligand with similar shape and coordination requirements to existing structural components, small energy differences between the two different linker analogues in the heterogeneous reaction create dynamic equilibrium necessary for interconversion.

A

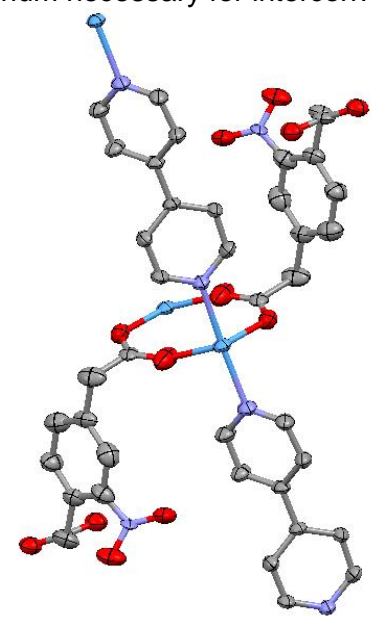

B

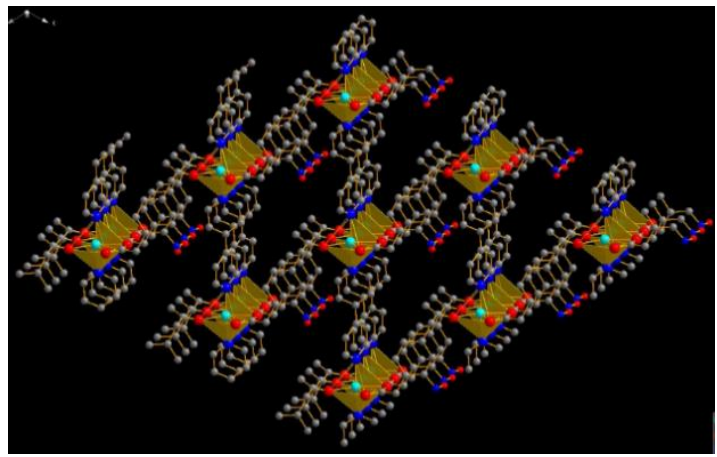

Figure 1. Thermal ellipsoid representation of NPDAC-MOF showing sppw structure with $50 \%$ thermal ellipsoids and labels for heteroatoms. Hydrogen atoms and $\mathrm{MeOH}$ are omitted for clarity. 1B. Two-dimensional layer showing the crystal packing, with bright yellow octahedral showing $\mathrm{Zn}^{2+}$ atoms are coordinated by four oxygen atoms and two nitrogen atoms.
A

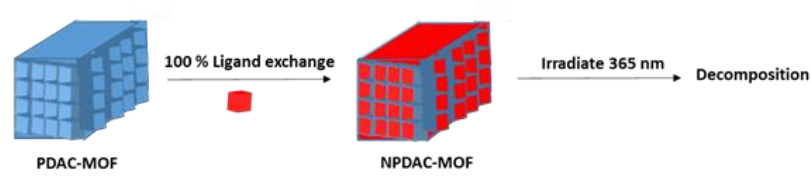

B

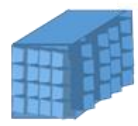

PDAC-MOF

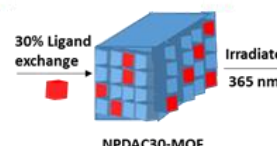

NPDAC30-MOF

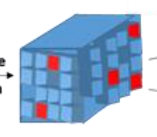

Defects forming
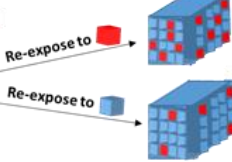

Scheme 2A. Full linker exchange reaction of PDAC-MOF with NPDAC to prepare NPDAC-MOF. NPDAC-MOF decomposes completely after $2 \mathrm{~h}$ when irradiated with $365 \mathrm{~nm}$ light. 2B. Partial linker exchange reaction used to prepare NPDAC30-MOF. Individual NPDAC units in NPDAC30-MOF undergo photodecarboxylation to provide intact MOFs with multiple defects. Exposure of photolyzed NPDAC30-MOF to either NPDAC or PDAC results in repair of the introduced defects.

Exposure of PDAC-MOF to a ten-fold excess of NPDAC with respect to PDAC resulted in the isolation colorless needles of NPDAC-MOF after 7 days (Scheme 2A). Given the unusual structure requirements of PDAC/NPDAC struts, we would not anticipate the PSLE process would result in well-defined materials when used in conjunction with other common MOF structural motifs. Single crystal $X$-ray analysis revealed nearly identical cell parameters for PDAC-MOF and NPDAC-MOF. Like PDAC-MOF the framework of NPDAC-MOF adopts a sppw structure (Figure 1A). Unlike typical pillared-paddle wheel structures, the two carboxylates are not in the same plane, forming a tilted paddle wheel with one NPDAC ligand facing up and the other facing down. An extended three-dimensional structure is shown in Figure 1B, with yellow octahedra representing $\mathrm{Zn}^{2+}$ atoms ligated by four oxygen and two nitrogen atoms. The nitro group on each dicarboxylate ligand is disordered in the X-ray structure; however, the digested ${ }^{1} \mathrm{H}$ NMR and LCMS experiments indicate the complete replacement of PDAC by NPDAC. The X-ray structure also reveals $\mathrm{MeOH}$ guest molecules contained within the NPDAC channels, which are quite narrow.

To understand how the linker exchange could take place in a PDAC-MOF that has limited space for diffusion into the coordination network, we performed quantum chemistry calculations on this structure. Upon removal of the encapsulated solvent molecules, we calculated the response of the resulting $P$ 1 layered structure to strain along the crystallographic a axis, which is perpendicular to its layers. The resulting energy profile is shown in Figure 2, and it clearly demonstrates the very "soft" nature of the PDAC-MOF along the a axis. Large variations of unit cell size are possible, up to $14 \%$ upon presence or absence of solvent molecules, with moderate energetic cost. Moreover, the smaller curvature of the energy profile at large a values, shows that the elastic modulus of the material in this region is small from a fit of that part of the curve, we can estimate a Young's modulus of $0.8 \mathrm{GPa}$, comparable to highly flexible framework materials. $\left.{ }^{51}, 52\right]$ This flexibility explains how linkers can easily 
diffuse in, through, and out of the MOF during the post-synthetic linker exchange process, even though based on purely geometric considerations of the experimental structure, diffusion of PDAC and NPDAC is expected to be very slow. This presence of "swollen" solvated phases in soft porous crystals, where diffusion is enhanced, has been demonstrated in several other materials in the past. $[53, \underline{54]}$

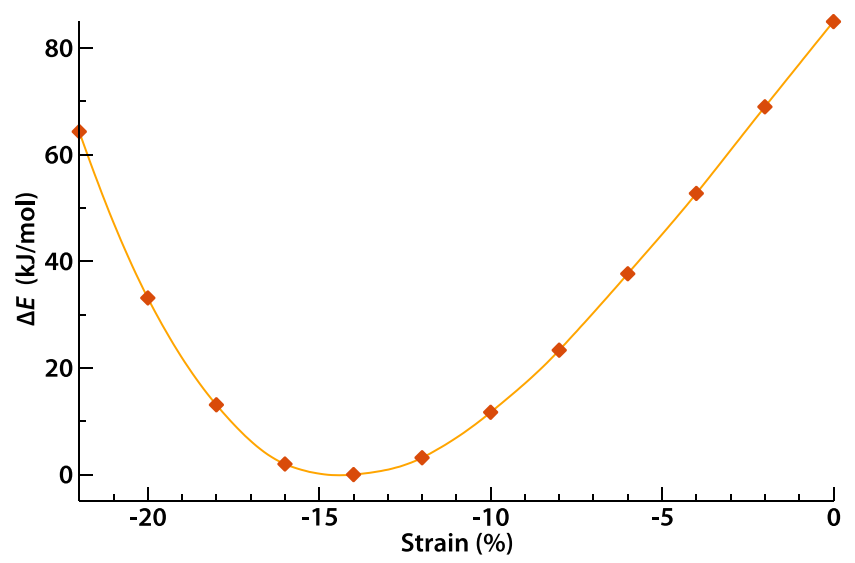

Figure 2. Variation of energy $\Delta E$ as a function of strain applied along the a axis of a PDAC-MOF, from DFT calculations. The strain is calculated with reference to the experimental PDAC-MOF cell parameters, and the lowest-energy structure (at strain $-14 \%$ ) is taken as energy reference.

The NPDAC-MOF obtained by PSLE appears to be stable indefinitely in the absence of light when stored in $\mathrm{MeOH}$. Upon exposure to UV light however, several photoproducts were detectable by UV-vis spectroscopy and LCMS (Figure 3). The increase in absorbance centered at $240 \mathrm{~nm}$ corresponds to 4,4'bipyridine, which is consistent with fragmentation of the paddle wheel sheets and release of the pillars. Like NPDAC ligand, three new peaks appear in the LC trace, corresponding to same three products of photodecarboxylation in addition to the 4,4'-bipyridine peak. Irradiation of PDAC-MOF under identical conditions reveals no evidence of decomposition. The photoreaction also can be monitored by PXRD. Initially, the NPDAC-MOF powder pattern simulated from using the single crystal data shows good agreement with predicted reflections. After $1 \mathrm{~h}$ of irradiation the disappearance of Bragg diffraction at $2 \theta$ between $7-13^{\circ}$ and 15$25^{\circ}$, along with the broadening of diffraction peaks, suggests decreasing crystallinity and increasing amorphization. All the Bragg diffraction peaks disappear after $2 \mathrm{~h}$ of irradiation, and two broad diffraction peaks at $2 \theta=5-10^{\circ}$ and $15-30^{\circ}$ appear, which indicates of complete phase change from crystalline to amorphous.

A light-digestible MOF has many possible intriguing applications such as a delivery vehicle for chemical reagents or therapeutic agents. Although the channels in NPDAC-MOF are relatively narrow, we have evidence that the material is flexible, so this may not necessarily preclude inclusion of medium-sized molecules. The necessity of using a NPAA group to achieve the photodecarboxylation currently limits the library of available MOF structures that can be rapidly accessed. Further development of our photodegradable MOF strategy will require exploring heretofore unknown structures prepared from new synthetic struts containing NPAA functional groups. Although we hope to expand the library of photodegradable MOF structures by developing a general design strategy for successfully using these flexible struts in the future, we sought to use the current model systems to explore the potential to semi-rationally modify the contents and properties of an assembled MOF.

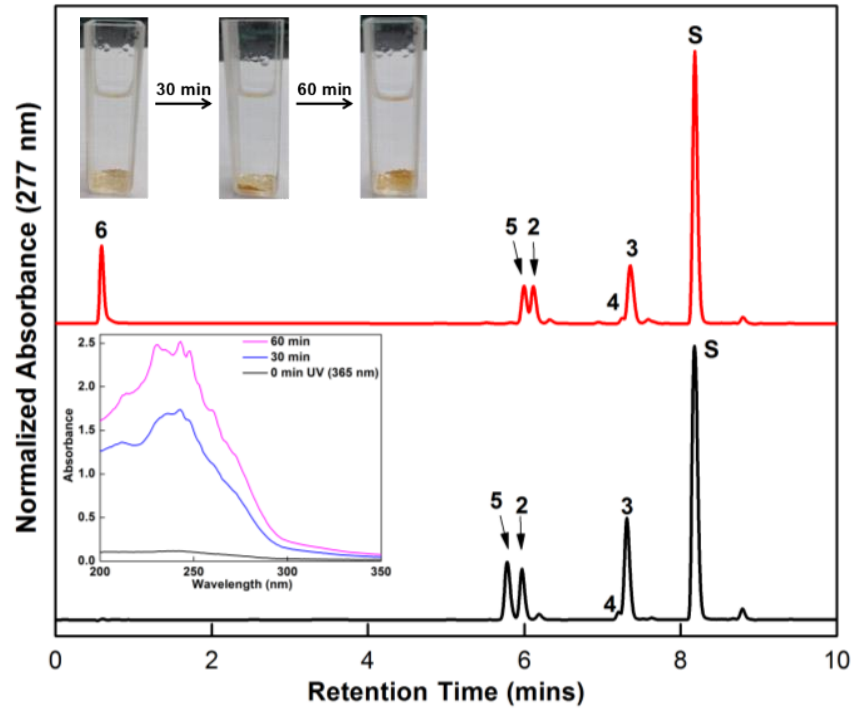

Figure 3. HPLC traces for the photolysis of NPDAC-MOF after $1 \mathrm{~h}$ (red) and NPDAC (2) after 10 min (black). Ketoprofen was used as internal standard (S) with 4-methyl-2-nitrophenylacetic acid (3), 2-nitro- $p$-xylene (4) and 4-methyl-2nitrobenzaldehyde (5) photoproducts, and liberated 4,4'-dipyridine (6). Upper inset photo shows the color changes in the crystalline material from light yellow to dark yellow over the course of the irradiation. Lower inset shows the changes in the UV-Vis spectrum of NPDAC-MOF after irradiating period of $1 \mathrm{~h}$, which indicates the presence of soluble nitro-phenyl species.

The original PSLE conditions used to prepare NPDAC-MOF suggested no incomplete strut replacement; however, reducing the excess of NPDAC in the PSLE reaction yields mixed PDAC/NPDAC MOFs. Specifically, by employing a 3-fold excess of NPDAC instead of a 10 -fold excess, the digested ${ }^{1} \mathrm{H}$ NMR suggested approximately $30 \%$ of PDAC in PDAC-MOF had been replaced by NPDAC (NPDAC30-MOF). We hypothesized that if a PDAC-MOF contained a sub-stoichiometic amount of a photodecomposable ligand, the reactive strut could be removed while retaining a stable or semi-stabile MOF structure. Furthermore, we would be able to observe evidence of vacancies with analytical techniques such as gas adsorption.

After extended irradiation of NPDAC30-MOF, no amorphous material was detected by PXRD; however, NMR analysis revealed the NPDAC content decreased from $30 \%$ to $16 \%$ after acid digestion. Currently, there does not appear to be a suitable analytical technique to map the NPDAC substitution pattern, or subsequent vacancies introduced within NPDAC30-MOF by 
photolysis. We suspect NPDAC groups nearer to the surface of the MOF are more efficiently removed since refracted light can be absorbed by bipyridine and PDAC groups within the lattice, thereby reducing the amount of light penetrating deep into the crystalline material capable of photolyzing internal NPDAC groups. The photolysis of NPDAC30-MOF resulted in a significant changes in the MOF surface area as measured by $\mathrm{N}_{2}$ adsorption using the BET method. NPDAC-MOF and PDAC-MOF exhibit nearly identical surface areas of 6.3 and $6.6 \mathrm{~m}^{2} / \mathrm{g}$, respectively. After photolysis however, the surface area of NPDAC-30 measured $99 \mathrm{~m}^{2} / \mathrm{g}$, an increase consistent with defects created within the MOF by strut photolysis and loss. Although photolysis of NPDAC30-MOF creates more surface area, the absolute value is over an order of magnitude smaller than that measured in many familiar MOFs.

The ability to create defects in MOF structures is of increasing interest, ${ }^{[5]}$ and there are a few examples of filling such vacancies by introducing additional struts. $\underline{56}, \underline{57]}$ To further explore the properties of the vacancies created in NPDAC30-MOF, an irradiated sample was divided into two equivalent portions, which were then immersed in solutions containing either 3-fold excess PDAC or NPDAC for $1 \mathrm{~d}$. The digested ${ }^{1} \mathrm{H}$ NMR shows the NPDAC content in the first portion is $32 \%$ and in the second portion is $13 \%$. These experiments suggest that a photolyzed NPDAC30-MOF can be efficiently reconstituted under facile conditions since the amount of NPDAC present after immersion is identical to that found in the original NPDAC30-MOF sample within experimental error ( $32 \%$ vs $30 \%)$. The decreased NPDAC (13\% vs $30 \%)$ and increased PDAC content after reconstituting the irradiated NPDAC30-MOF with PDAC further supports the reconstitution model (Scheme 2B).

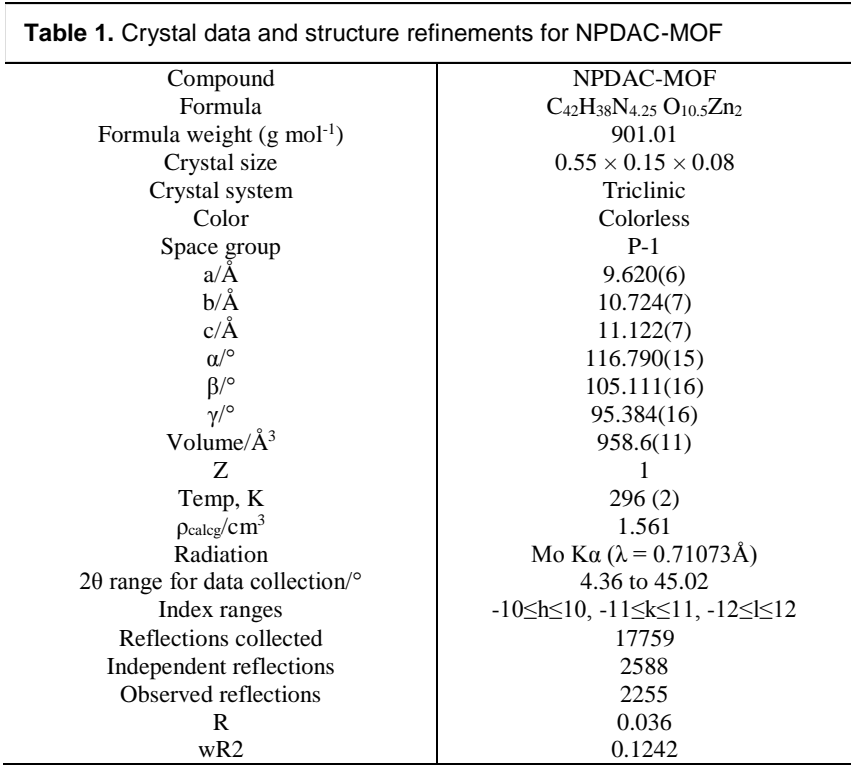

The NPDAC percentage in both portions of the reconstitution experiment corresponds to the theoretical content if all the defect sites were filled by one of the two ligands. Since the reconstitution process is significantly faster than the original PSLE synthesis, we hypothesize that the MOF structural flexibility provides ready access to the vacancies created by photoysis. Although the filling of open sites should be faster, we cannot yet rule out the possibility of substitution reactions at intact sites.

\section{Conclusions}

Utilizing a photolabile ligand as MOF strut can make a framework undergo full or partial decomposition upon irradiation. A NPAA derivative has been incorporated into MOF as a backbone linker via PLSE method. The photo-induced decarboxylation of the NPDAC-MOF represents a novel way of degrading a MOF, which provides an innovative approach to formulating photoresponsive porous materials with potential applications ranging from molecular release to drug delivery. When photoactive linker is mixed with non-photolabile linker via partial PLSE, the MOF structure can be retained after irradiation, but with the introduction of multiple vacancies, offering a new method to create defects in MOFs. Defect repair can be achieved by treatment with replacement ligands, the scope of which is an interesting area for developing customizable MOF contents. The use of carboxylate struts capable of undergoing photodecarboxylation may lead to a general approach introducing MOF defects for a variety of new applications, and will be the subject of further investigations.

\section{Acknowledgements}

This work was supported by the National Science Foundation grant CHE-0955361, the American Chemical Society Petroleum Research Fund grant 53977-ND3 and Worcester Polytechnic Institute. Access to high-performance computing platforms was provided by GENCI grant A0050807069.

Keywords: Metal Organic Framework • Photodecomposition • Ligand Exchange Reactions $\cdot$ Density Functional Theory $\bullet X$-ray Crystallography

[1] J. Ren, H. W. Langmi, B. C. North, M. Mathe, Int. J. Energy Res. 2015 39, 607-620.

[2] P. Puthiaraj, Y.-R. Lee, S. Ravi, S. Zhang, W.-S. Ahn, in Postcombustion Carbon Dioxide Capture Materials, The Royal Society of Chemistry, 2019, pp. 153-205.

[3] S. Qiu, M. Xue, G. Zhu, Chem. Soc. Rev. 2014, 43, 6116-6140.

[4] F.-X. Coudert, Chem. Mater. 2015, 27, 1905-1916.

[5] J. Park, D. Q. Yuan, K. T. Pham, J. R. Li, A. Yakovenko, H. C. Zhou, J. Am. Chem. Soc. 2012, 134, 99-102.

[6] R. Lyndon, K. Konstas, B. P. Ladewig, P. D. Southon, C. J. Kepert, M. R Hill, Angew. Chem. Int. Ed. Engl. 2013, 52, 3695-3698.

[7] J. Zhang, L. B. Wang, N. Li, J. F. Liu, W. Zhang, Z. B. Zhang, N. C. Zhou, X. L. Zhu, CrystEngComm. 2014, 16, 6547-6551.

[8] J. Yan, L. Wilbraham, P. N. Basa, M. Shuettel, J. C. MacDonald, I. Ciofini, F. X. Coudert, S. C. Burdette, Inorg. Chem. 2018, 57, 1500915022.

[9] J. Yan, A. D. Carl, J. C. MacDonald, P. Müller, R. L. Grimm, S. C. Burdette, Dalton Trans. 2019, accepted.

[10] C. B. Fan, L. Le Gong, L. Huang, F. Luo, R. Krishna, X. F. Yi, A. M. Zheng, L. Zhang, S. Z. Pu, X. F. Feng, M. B. Luo, G. C. Guo, Angew. Chem. Int. Ed. Engl. 2017, 56, 7900-7906.

[11] F. Luo, C. B. Fan, M. B. Luo, X. L. Wu, Y. Zhu, S. Z. Pu, W. Y. Xu, G. C Guo, Angew. Chem. Int. Ed. Engl. 2014, 53, 9298-9301. 
[12] I. M. Walton, J. M. Cox, C. A. Benson, D. G. Patel, Y. S. Chen, J. B. Benedict, New J. Chem. 2016, 40, 101-106.

[13] R. K. Deshpande, G. I. N. Waterhouse, G. B. Jameson, S. G. Telfer Chem. Commun. 2012, 48, 1574-1576.

[14] C. A. Allen, S. M. Cohen, J. Mater. Chem. 2012, 22, 10188-10194.

[15] K. M. L. Taylor-Pashow, J. Della Rocca, Z. G. Xie, S. Tran, W. B. Lin, J. Am. Chem. Soc. 2009, 131, 14261-14263.

[16] C. Orellana-Tavra, S. Haddad, R. J. Marshall, I. A. Lazaro, G. Boix, I. Imaz, D. Maspoch, R. S. Forgan, D. Fairen-Jimenez, ACS Appl. Mater. Inter. 2017, 9, 35516-35525.

[17] I. A. Lazaro, S. Haddad, S. Sacca, C. Orellana-Tavra, D. FairenJimenez, R. S. Forgan, Chem-Us 2017, 2, 561-578.

[18] J. Xiang, F. J. Ge, B. Yu, Q. Yan, F. Shi, Y. Zhao, ACS Appl. Mater. Inter. 2018, 10, 20790-20800.

[19] T. C. Zhao, P. Y. Wang, Q. Li, A. A. Al-Khalaf, W. N. Hozzein, F. Zhang, X. M. Li, D. Y. Zhao, Angew. Chem. Int. Ed. Engl. 2018, 57, 2611-2615.

[20] G. Liu, C. M. Dong, Biomacromolecules 2012, 13, 1573-1583.

[21] P. Klan, T. Solomek, C. G. Bochet, A. Blanc, R. Givens, M. Rubina, V. Popik, A. Kostikov, J. Wirz, Chem. Rev. 2013, 113, 119-191.

[22] H. W. Mbatia, D. P. Kennedy, S. C. Burdette, Photochem. Photobiol. 2012, 88, 844-850.

[23] P. N. Basa, S. Antala, R. E. Dempski, S. C. Burdette, Angew. Chem. Int. Ed. Engl. 2015, 54, 13027-13031.

[24] X. Han, Q. Cheng, X. R. Meng, Z. C. Shao, K. Ma, D. H. Wei, J. Ding, H. W. Hou, Chem. Commun. 2017, 53, 10314-10317.

[25] C. C. Epley, K. L. Roth, S. Y. Lin, S. R. Ahrenholtz, T. Z. Grove, A. J. Morris, Dalton Trans. 2017, 46, 4917-4922.

[26] J. Yan, R. Homan, C. Boucher, P. N. Basa, R. L. Grimm, J. C. MacDonald, S. C. Burdette, in preparation 2018.

[27] T. F. Liu, J. Lu, L. X. Shi, Z. G. Guo, R. Cao, CrystEngComm. 2009, 11, 583-588.

[28] G. M. Sheldrick, University of Göttingen: Göttingen, Germany, 1996.

[29] G. M. Sheldrick, Acta Crystallogr. C 2015, 71, 3-8.

[30] J. E. Jaffe, A. C. Hess, Phys. Rev. B 1993, 48, 7903-7909.

[31] M. A. Spackman, A. S. Mitchell, Phys. Chem. Chem. Phys. 2001, 3, 1518-1523.

[32] R. Dovesi, R. Orlando, A. Erba, C. M. Zicovich - Wilson, B. Civalleri, S. Casassa, L. Maschio, M. Ferrabone, M. De La Pierre, P. D'Arco, Int. J. Quantum Chem. 2014, 114, 1287-1317.

[33] J. P. Perdew, A. Ruzsinszky, G. I. Csonka, O. A. Vydrov, G. E. Scuseria . A. Constantin, X. Zhou, K. Burke, Phys. Rev. Lett. 2008, 100, 136406.

[34] S. Grimme, J. Antony, S. Ehrlich, H. Krieg, J. Chem. Phys. 2010, 132, 154104.

[35] G. P. Yang, Y. Y. Wang, W. H. Zhang, A. Y. Fu, R. T. Liu, E. K. Lermontova, Q. Z Shi, CrystEngComm. 2010, 12, 1509-1517.
[36] G. G. Sezer, O. Z. Yesilel, H. Erer, O. Sahin, J. Solid State Chem. 2016, 233, 463-470.

[37] J. D. P. Margerum, C. T., J. Am. Chem. Soc. 1969, 91, 2467-2472.

[38] J. M. Mewes, K. Neumann, M. K. Verhoefen, G. Wille, J. Wachtveitl, A Dreuw, ChemPhysChem. 2011, 12, 2077-2080.

[39] R. Lechner, S. Kummel, B. Konig, Photochem. Photobiol. Sci. 2010, 9, 1367-1377.

[40] A. Itoh, T. Kodama, S. Inagaki, Y. Masaki, Org. Lett. 2000, 2, 331-333.

[41] Y. Maki, M. Sako, I. Oyabu, T. Murase, Y. Kitade, K. Hirota, Chem. Commun. 1989, 1780-1782.

[42] H. Koshima, Mol. Cryst. Liq. Cryst. 2005, 440, 207-214

[43] Y. Wei, P. Hu, M. Zhang, W. P. Su, Chem. Rev. 2017, 117, 8864-8907.

[44] H. T. Song, W. Ding, Q. Q. Zhou, J. Liu, L. Q. Lu, W. J. Xiao, J. Org Chem. 2016, 81, 7250-7255.

[45] Q. Feng, Q. L. Song, J. Org. Chem. 2014, 79, 1867-1871.

[46] O. Karagiaridi, W. Bury, J. E. Mondloch, J. T. Hupp, O. K. Farha, Angew. Chem. Int. Ed. Engl. 2014, 53, 4530-4540.

[47] K. K. Tanabe, S. M. Cohen, Angew. Chem. Int. Ed. Engl. 2009, 48 7424-7427.

[48] H. H. Fei, J. F. Cahill, K. A. Prather, S. M. Cohen, Inorg. Chem. 2013 52, 4011-4016.

[49] W. Bury, D. Fairen-Jimenez, M. B. Lalonde, R. Q. Snurr, O. K. Farha, J. T. Hupp, Chem. Mater. 2013, 25, 739-744.

[50] K. L. Mulfort, O. K. Farha, C. L. Stern, A. A. Sarjeant, J. T. Hupp, J. Am Chem. Soc. 2009, 131, 3866-3868.

[51] S. Horike, S. Shimomura, S. Kitagawa, Nat. Chem. 2009, 1, 695

[52] J.-C. Tan, B. Civalleri, C.-C. Lin, L. Valenzano, R. Galvelis, P.-F. Chen, T. D. Bennett, C. Mellot-Draznieks, C. M. Zicovich-Wilson, A. K. Cheetham, Phys. Rev. Lett. 2012, 108, 095502.

[53] A. Boutin, D. Bousquet, A. I. U. Ortiz, F. o.-X. Coudert, A. H. Fuchs, A. Ballandras, G. Weber, I. Bezverkhyy, J.-P. Bellat, G. Ortiz, J. Phys. Chem. C 2013, 117, 8180-8188.

[54] F. o.-X. Coudert, A. I. U. Ortiz, V. Haigis, D. Bousquet, A. H. Fuchs, A Ballandras, G. Weber, I. Bezverkhyy, N. Geoffroy, J.-P. Bellat, J. Phys Chem. C 2014, 118, 5397-5405

[55] Z. L. Fang, B. Bueken, D. E. De Vos, R. A. Fischer, Angew. Chem. Int Ed. Engl. 2015, 54, 7234-7254.

[56] O. V. Gutov, M. G. Hevia, E. C. Escudero-Adan, A. Shafir, Inorg. Chem. 2015, 54, 8396-8400

[57] X. R. Wang, L. Z. Zhai, Y. X. Wang, R. T. Li, X. H. Gu, Y. Di Yuan, Y. H. Qian, Z. G. Hu, D. Zhao, ACS Appl. Mater. Inter. 2017, 9, 37848-37855. 